\title{
Identification of Temperature-Dependent Model Parameters of Ultrasonic Piezo-Composite Transducers
}

\author{
Manuel Webersen ${ }^{1}$, Fabian Bause ${ }^{1}$, Jens Rautenberg ${ }^{1}$, Bernd Henning ${ }^{1}$ \\ ${ }^{1}$ Measurement Engineering Group, University of Paderborn, Warburger Str. 100, Paderborn, Germany \\ webersen@emt.uni-paderborn.de
}

\begin{abstract}
:
When performing measurements, the effects of the measurement system itself on the measured data generally must be eliminated. Consequently, those effects, i.e. the system's dynamic behavior, need to be known. For the piezo-composite transducers in an ultrasonic transmission line, a model based approach is used to describe their dynamic behavior and take into account its dependence on the environment temperature and the acoustic impedance of the target medium.

Temperature-dependent model parameters are presented, which are obtained by performing a multiplepart identification process on the transducer model, based on electrical impedance measurements [1]. The identification process uses an inverse approach for optimizing a subset of the model parameters. Additionally, algorithmic differentiation methods are used to determine accurate derivatives.

In a final optimization step, impedance measurements taken at different temperatures are used to determine the temperature dependencies of the model parameters. These can then be used to assess the plausibility of the identification results. Additionally, the parameters can be expressed as polynomials in the temperature to take different operating conditions into account.
\end{abstract}

Key words: piezo-composite, transducer, temperature dependency, identification, plausibility

\section{Introduction}

An ultrasonic transmission line is used to determine material properties of a sample under test. For this, the impulse response must be calculated based on the electric received signal with respect to the electric send signal. The received signal, however, also includes influences caused by the measurement setup, i.e. the ultrasonic transducers as well as sending and receiving amplifiers. So, to be able to determine the impulse response of only the sample, the impulse responses of the aforementioned system components must be known.

The transducer's dynamic behavior strongly depends on the environment temperature and the acoustic impedance of the target medium (i.e. the sample). As the system is explicitly intended to be used with different samples and different temperatures, a simple referencing technique is insufficient for eliminating the transducer's effect on the received signal. Instead, a model based approach is chosen, so that the transducer's dynamic behavior may be simulated for any (feasible) operating condition.

This in turn requires the identification of model parameters, such that the model describes a real transducer as accurately as possible. For parameters that change significantly over temperature, the temperature dependence must be characterized additionally.

\section{Transducer design}

The transducers in use include a Sonotec 1-3 piezo-composite disk as the active element, allowing one-dimensional modeling. Passive components include an adhesive layer and a stainless steel protection layer on the front and a damping mass on the back. The backing material is a mixture of polyurethane and tungsten carbide. The design also includes a steel sleeve to hold the backing material as well as a plastic housing. Fig. 1 shows the basic setup (housing omitted).

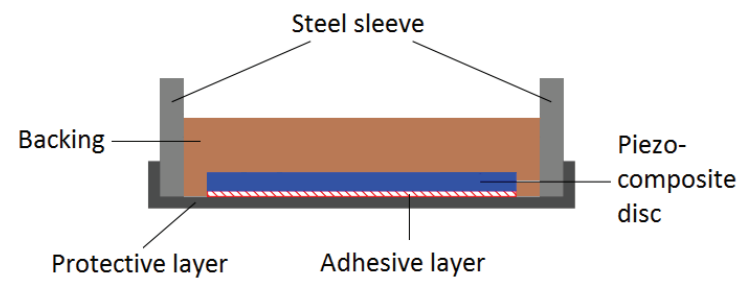

Fig. 1. Sectional drawing of the transducer 


\section{Transducer model}

The transducer model is based on the Mason model $[2,3]$, which describes the active element as a three-port with an electric and two mechanic I acoustic ports. This allows to add electric networks or mechanic layers (here: adhesive and protection) by describing them as two-ports.

In sending mode, the electric port is used as the input and the branch corresponding to the front side of the transducer (i.e. the side facing the target medium) is terminated with the acoustic impedance of the target medium $Z_{\mathrm{T}}$. In receiving mode, the front acoustic port is used as the input, and the electric port is terminated with the input impedance of the receiving amplifier $Z_{\mathrm{i}}$. In both cases, the rear acoustic port is terminated with the backing impedance $Z_{\mathrm{B}}$. Figs. 2 and 3 show the network diagram for both modes, with all electric and mechanic two-ports condensed into $A_{\text {el }}$ and $A_{\mathrm{m}}$, respectively.

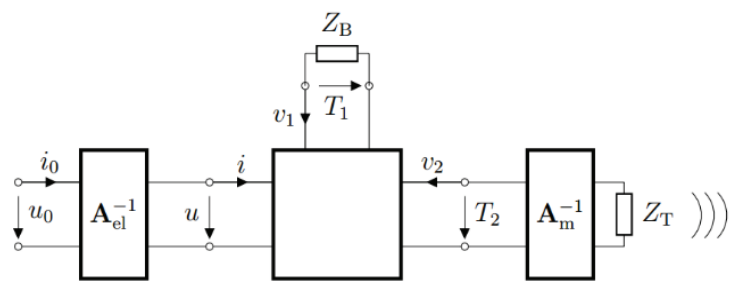

Fig. 2. Extended Mason model (sending mode)

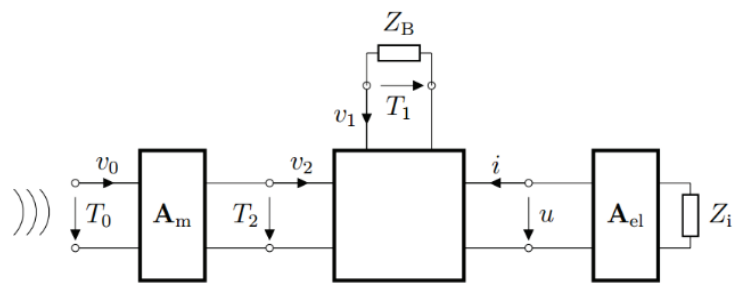

Fig. 3. Extended Mason model (receiving mode)

For the three-port describing the piezocomposite disk, the following model parameters must be defined:

- Thickness $t$

- $\quad$ Diameter $D$

- $\quad$ Mass $m$

- Density $\rho$

- Sound velocity $c_{\mathrm{T}}^{D}$ (thickness mode, constant electric displacement field)

- $\quad$ Electro-mechanic coupling factor $k_{\mathrm{T}}$

- Mechanical vibration quality $Q_{\mathrm{m}}$

- Permittivity $\varepsilon_{33}^{S}$ (3-direction, constant strain)

- Density of electrode material $\rho_{\mathrm{e}}$

- $\quad$ Sound velocity in electrode material $c_{\mathrm{e}}$
For the assembled transducer, the adhesive and protection layers are added as two-ports, plus the acoustic impedance of the backing material. Consequently, the following parameters are added to the model:

- Thickness of the adhesive layer $t_{\mathrm{adh}}$

- Density of the adhesive layer $\rho_{\text {adh }}$

- Sound velocity of the adhesive layer $c_{\text {adh }}$

- Thickness of the protective layer $t_{\text {prot }}$

- Density of the protective layer $\rho_{\text {prot }}$

- Sound velocity of the protective layer $c_{\text {prot }}$

- Acoustic impedance of the backing material $Z_{\mathrm{B}}$

Additionally, two-port models for different types of electric networks (using e.g. $\mathrm{T}$ - and $\mathrm{Pi}$ networks or the telegraph equations) are available with their respective parameters.

It should be noted that all model parameters may be specified as polynomials in the temperature, so that temperature dependence can be represented.

\section{Nonlinear inverse parameter identification}

When using the transducer model to describe a specific instance of actual, physical transducer, the model parameters must be chosen such that the modeled behavior matches the real behavior as accurately as possible. This presents an inverse problem, because the model calculates the system output $\mathbf{y}$ (i.e. effect) for given inputs $\Theta$ (i.e. causes), but in contrast, the identification requires the input to be concluded from the output. However, if the model is not invertible, an analytic solution for the model parameters may not be obtainable. Then, specific approaches to inverse problems may be applied [4].

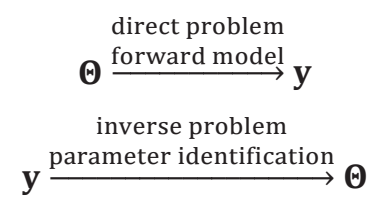

Indeed, the model does not allow a direct solution, so the approach depicted in fig. 4 is used. Starting with an estimation of the desired parameters, the model output $\mathbf{y}$ is calculated and compared to actual measurement data d via a cost function. In an iterative process, an optimizer then varies selected model parameters until the cost function reaches its minimum. This setup may also be regarded as an adaptive filter for system identification (albeit using a more complex cost function to determine $\xi$ ). 


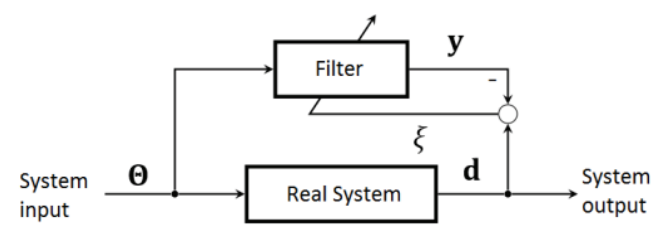

Fig. 4. Adaptive filter for system identification (adapted from [5])

Both the forward model and the parameter identification are implemented in MATLAB. For the optimization, MATLAB's fmincon function is used with the interior-point algorithm. However, instead of applying the internal finite differences (FD) method to approximate the required derivatives (i.e. Jacobians), the option of supplying externally calculated derivatives is used.

To determine those Jacobians, ADiMat [6], a MATLAB library that implements algorithmic differentiation (AD) techniques, is used. In contrast to finite differences, $A D$ provides exact derivatives (to machine precision), thus eliminating both numerical errors in subsequent calculations and possible problems caused by inappropriate parameterization (e.g. FD step size). It is worth noting that $F D$ and $A D$ require similar amounts of computation time, so using $A D$ does not introduce a significant speed penalty.

\section{Identification process}

The identification process operates on the frequency dependent electric impedance of the transducer. The complex physical quantity $Z_{\text {meas }}(\mathrm{j} \omega)$ is measured using a network analyzer (Anritsu MS4630B) and the corresponding model output $Z_{\text {sim }}(\mathrm{j} \omega)$ is simulated.

For the complete identification, the inverse approach described above is applied in multiple instances. Individual instances differ in the subset of model parameters to be optimized and in the form of the cost function. By only optimizing few parameters at a time, the complexity of the inverse problem and thus the ambiguity of the results can be reduced drastically. Often, without this kind of reduction, no plausible results can be obtained at all.

Because of this, the identification process is divided into three parts which successively introduce more complexity to the model:

1. Identification of piezo-composite disk

2. Identification of assembled transducer at room temperature

3. Identification of the temperature dependence
Below, these parts are described in detail. However, before any optimizations can be performed, initial values for all model parameters (in the current setup) must be defined. Quantities that cannot be measured directly must be estimated from other model parameters or gathered from literature or datasheets. For the piezo-composite disk, this has already been described in [1] and is not discussed further in this contribution.

\section{Identification of the piezo-composite disk}

For the piezo-composite disk, the following five model parameters are optimized:

- Density $\rho$

- $\quad$ Sound velocity $c_{\mathrm{T}}^{D}$

- Coupling factor $k_{\mathrm{T}}$

- Mechanical vibration quality $Q_{\mathrm{m}}$

- Permittivity $\varepsilon_{33}^{S}$

Other parameters, e.g. the thickness, diameter and mass of the composite disk, are not optimized because they can be measured with negligible measurement uncertainty.

The main difficulty is caused by strong cross sensitivities of the parameters, e.g. sound velocity and coupling factor, which prevent the optimizer from reaching a (physically plausible) minimum of the cost function.

However, the influence of the model parameters on the electric impedance (and thus on the cost function) strongly depends on the frequency, and this dependence differs for the individual parameters: While $\rho, c_{\mathrm{T}}^{D}$ and $Q_{\mathrm{m}}$ are sensitive in the vicinity of both the resonance and antiresonance frequencies $\left(f_{\mathrm{r}} \cong 1.03 \mathrm{MHz}\right.$ and $\left.f_{\mathrm{a}} \cong 1.28 \mathrm{MHz}\right), k_{\mathrm{T}}$ is only sensitive near the resonance frequency. The sensitivity on $\varepsilon_{33}^{S}$ is actually independent of the frequency. Figs. 5 through 8 show the respective frequencydependent sensitivities.

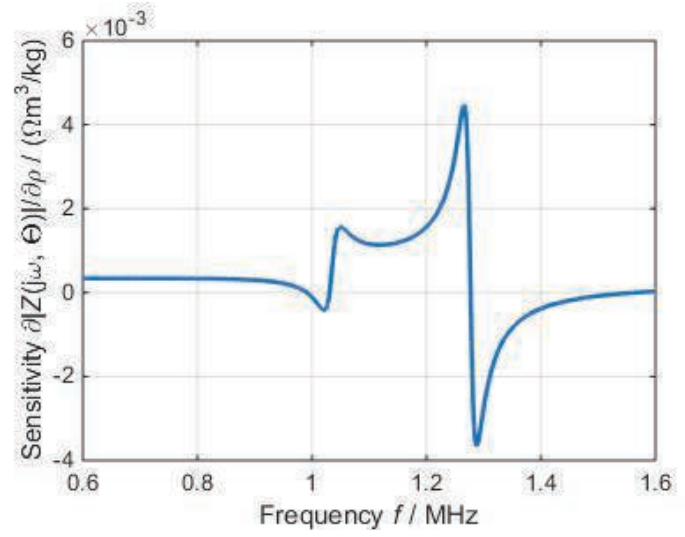

Fig. 5. Sensitivity w.r.t. $\rho$ 


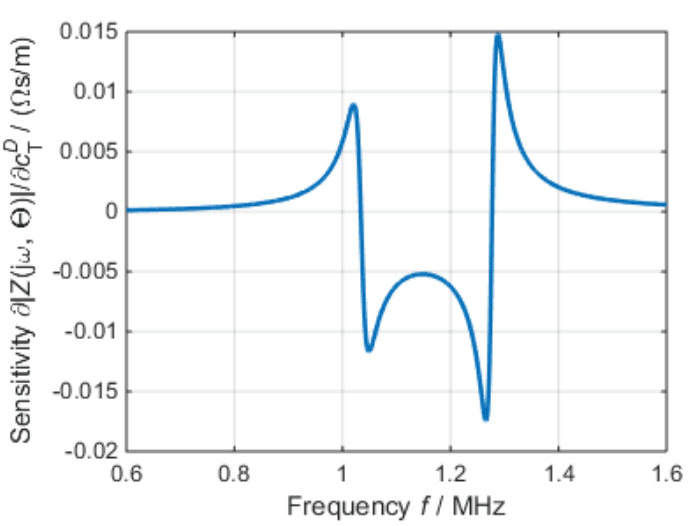

Fig. 6. Sensitivity w.r.t. $c_{\mathrm{T}}^{D}$

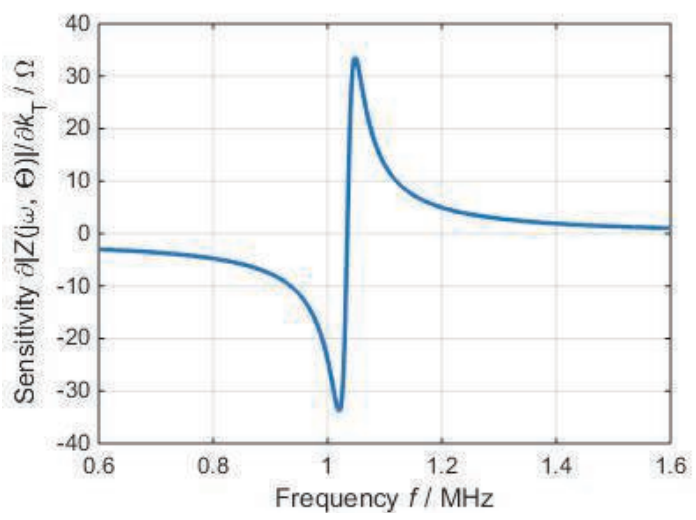

Fig. 7. Sensitivity w.r.t. $k_{\mathrm{T}}$

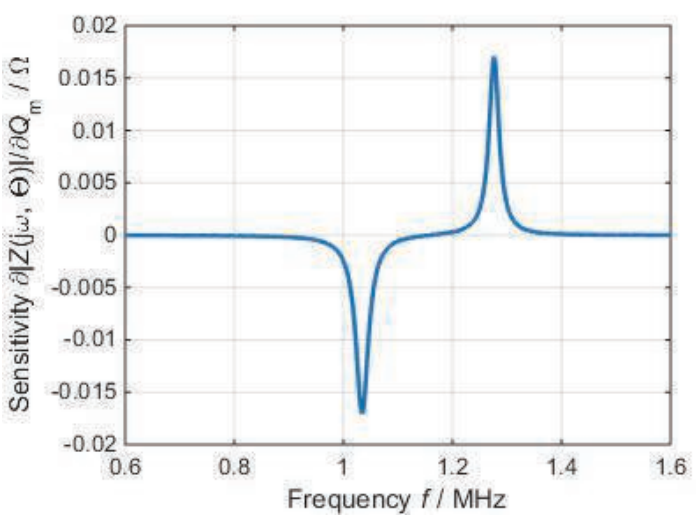

Fig. 8. Sensitivity w.r.t. $Q_{\mathrm{m}}$

This frequency dependence allows to decouple the cross sensitivities by splitting the identification into four optimization steps. Each step optimizes a subset of the model parameters and uses an adjusted cost function $\xi_{i}$ (via the frequency-dependent weighting function $g_{i}(\omega)$ ) to select only the frequency ranges needed for the current parameters. The weighting function uses a Blackman window to select or unselect a band of $200 \mathrm{kHz}$ around the resonance and/or antiresonance frequency. Eq. (1) shows the cost function for step $i$, expressed for continuous angular frequencies $\omega$. Evaluation in MATLAB uses a discretized form.

$$
\begin{gathered}
\xi_{i}(\boldsymbol{\Theta})=\int\left(\left(\ln \left|Z_{\operatorname{sim}}(\mathrm{j} \omega, \boldsymbol{\Theta})\right|-\ln \left|Z_{\text {meas }}(\mathrm{j} \omega)\right|\right)\right. \\
\left.\cdot g_{i}(\omega)\right)^{2} \mathrm{~d} \omega
\end{gathered}
$$

Table 1 shows the configuration of the optimization steps.

Tab. 1: Identification steps for piezo-composite disk

\begin{tabular}{|c|c|c|}
\hline$\#$ & Parameters & Weighting \\
\hline 1 & $\varepsilon_{33}^{S}$ & outside $f_{\mathrm{r}}, f_{\mathrm{a}}$ \\
\hline 2 & $k_{\mathrm{T}}$ & only $f_{\mathrm{r}}$ \\
\hline 3 & $\rho, c_{\mathrm{T}}^{D}, Q_{\mathrm{m}}$ & $f_{\mathrm{r}}$ and $f_{\mathrm{a}}$ \\
\hline 4 & $\rho, c_{\mathrm{T}}^{D}, Q_{\mathrm{m}}, \varepsilon_{33}^{S}$ & $f_{\mathrm{a}}$ \\
\hline
\end{tabular}

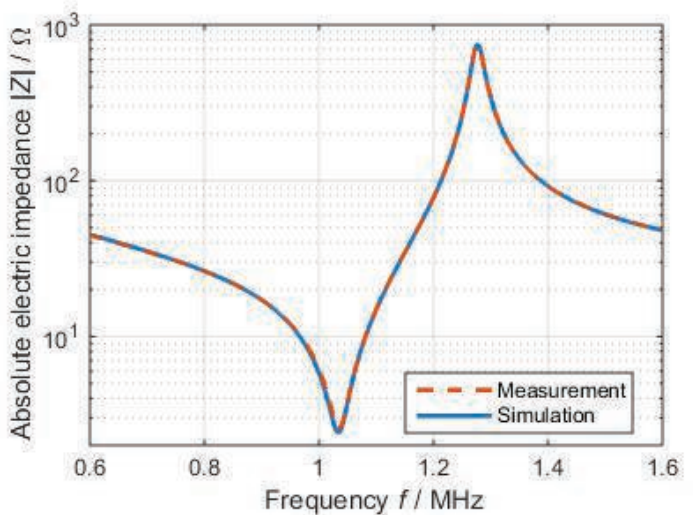

Fig. 9. Identification of the piezo-composite disk

Fig. 9 shows the measured and simulated absolute electric impedance after the identification.

\section{Assembled transducer}

For the assembled transducer, the model of the piezo-composite disk (with previously identified parameters) is extended by two acoustic layers on the front, representing the adhesive and protective layers, as well as the backing on the rear side. This introduces seven new parameters to the overall model $\left(t_{\mathrm{adh}}, \rho_{\mathrm{adh}}, c_{\mathrm{adh}}, t_{\mathrm{prot}}, \rho_{\mathrm{prot}}\right.$, $c_{\text {prot }}$ and $Z_{\mathrm{B}}$ ).

However, not all of these parameters are optimized. First, just like the piezo-composite disk, the thickness of both layers can be measured directly and accurately, so no further optimization is required. The other four layer parameters are taken from literature and should be considered uncertain, but an optimization has been turned out to be not practical. Because the transducer's dynamic behavior depends on the reflection coefficient at the layer boundaries, which itself depends on the layer's acoustic impedance $Z_{\mathrm{ac}}=\rho c$, a change in any of the four 
parameters has the same basic effect on the electric impedance. The effect is also very similar to the one caused by a change of $c_{\mathrm{T}}^{D}$. Consequently, the solution would be highly ambiguous and not physically plausible. Additionally, the sensitivity of the electric impedance w.r.t. the layer parameters is small, so a significant change in the impedance requires a substantial (and again physically implausible) change in the layer parameters.

For a practical solution, only one of the affected parameters can be optimized. Due to the significantly higher sensitivity of $c_{\mathrm{T}}^{D}$ compared to the layer parameters, $c_{\mathrm{T}}^{D}$ is used in the optimization. However, the resulting values must be interpreted with caution: Any deviation of the layer parameters from the (unknown) real material properties is not corrected by the optimization, but transformed into a change of $c_{\mathrm{T}}^{D}$ instead.

In addition to $c_{\mathrm{T}}^{D}$, two more parameters associated with the piezo-composite disk are optimized: For simplicity, instead of introducing additional capacities, the permittivity $\varepsilon_{33}^{S}$ is readjusted to represent scattering fields between the rear electrode of the piezo-composite disk and the steel sleeve (which is connected to the front electrode). On the other hand, the coupling factor $k_{\mathrm{T}}$ is adjusted because the the resonance frequency changes during the transducer assembly. This change is likely caused by the temperature treatment processes that are used for setting the adhesive and backing material (45 minutes at $80^{\circ} \mathrm{C}$ and $75^{\circ} \mathrm{C}$, respectively).

Finally, the acoustic backing impedance $Z_{\mathrm{B}}$ is also optimized, as it can only be estimated based on the mass ratio of the two components [1].

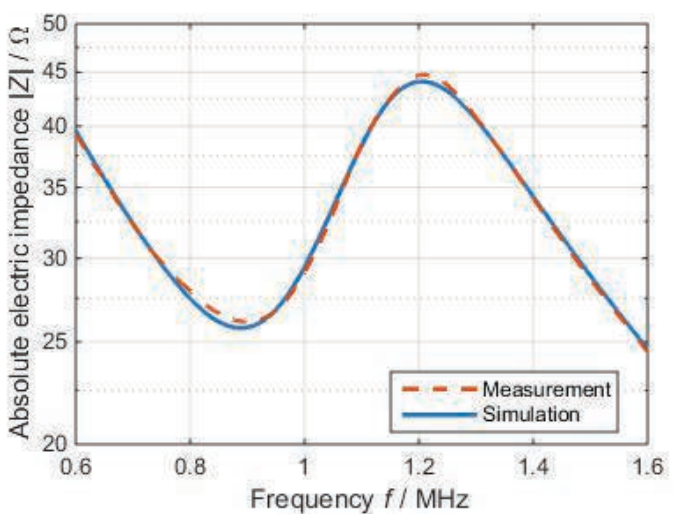

Fig. 10. Identification of the assembled transducer

The optimization is performed in one step and without any weighting applied to the cost function. Fig. 10 shows the measured and simulated absolute electric impedance after the identification of the assembled transducer.

\section{Temperature dependence}

In the third part of the identification process, the temperature dependence of model parameters is determined. For this, electric impedance measurements taken at different temperatures $\left(20^{\circ} \mathrm{C} \ldots 50^{\circ} \mathrm{C}\right.$, steps of $\left.5 \mathrm{~K}\right)$ are used in multiple successive identification steps.

The identification steps use the same structure and optimization parameters as in the previous part (i.e. $c_{\mathrm{T}}^{D}, k_{\mathrm{T}}, \varepsilon_{33}^{S}$ and $Z_{\mathrm{B}}$ ). However, as the expected relative change in value from one temperature to the next is small, the upper and lower bounds for the optimization can be adjusted. This requires the identification steps to be executed ordered by temperature, but reduces the chance of receiving physically implausible results.

The result is a temperature dependent sequence of values for each of the optimized model parameters, which is then approximated by a polynomial of degree 2. Afterwards, the model can be applied to arbitrary (feasible) temperatures.

Figs. 11 through 14 show the identification results as well as polynomial approximation for all of the optimized parameters.

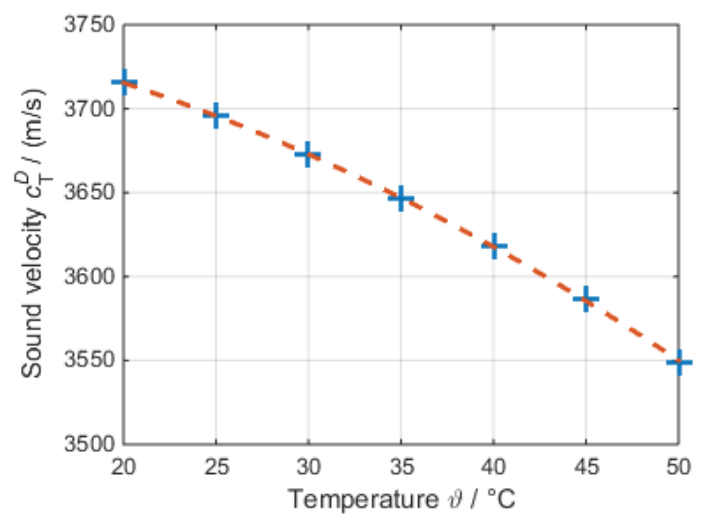

Fig. 11. Temperature dependence of $c_{\mathrm{T}}^{D}$

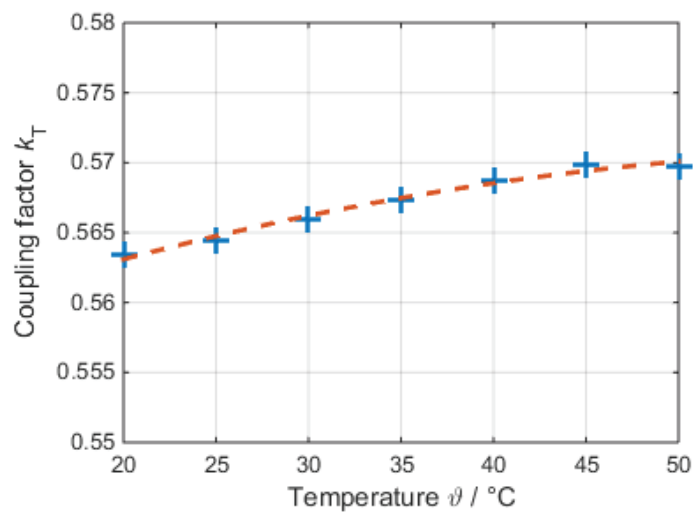

Fig. 12. Temperature dependence of $k_{\mathrm{T}}$ 


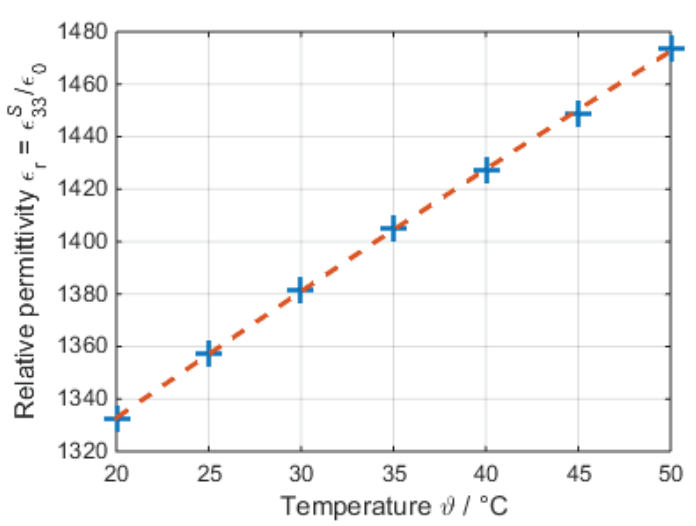

Fig. 13. Temperature dependence of $\varepsilon_{\mathrm{r}}=\frac{\varepsilon_{33}^{S}}{\varepsilon_{0}}$

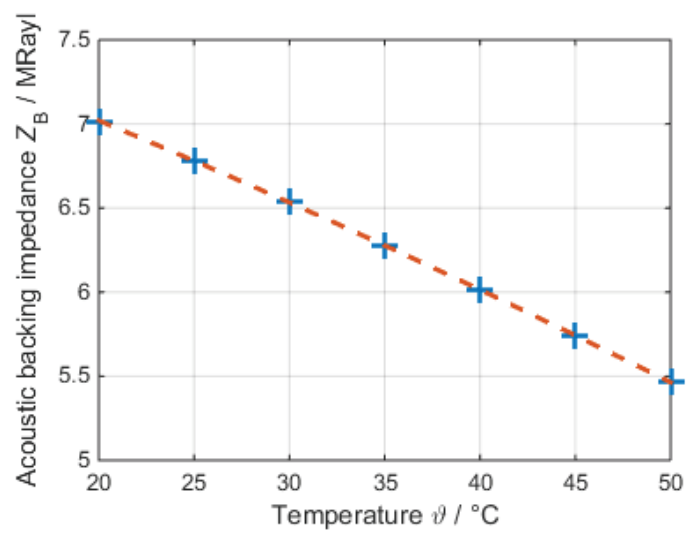

Fig. 14. Temperature dependence of $Z_{\mathrm{B}}$

\section{Evaluation}

As shown by figures 9 and 10 , both the piezocomposite disk and the assembled transducer can be modeled accurately. The maximum deviation from the measurement data, occurring near the antiresonance frequency, is $5 \Omega(0.7 \%)$ and $0.7 \Omega(1.6 \%)$, respectively. Even after the identification of the temperature dependence (see fig. 15), the deviation never exceeds $1 \Omega$ for any of the transducers. This deviation is close to the measurement uncertainty specified by Anritsu, which suggests a successful identification.

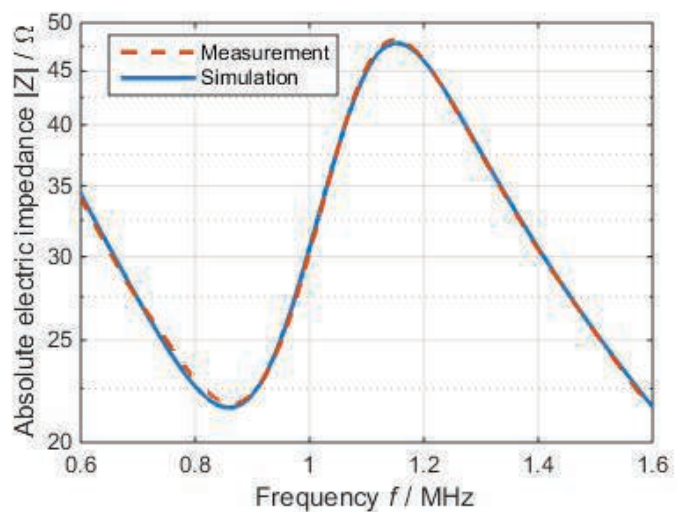

Fig. 15. Fully identified transducer (at $50^{\circ} \mathrm{C}$ )
Additionally, the systematic behavior of the identified model parameters over temperature (see figs. 11-14) suggests that its cause indeed is the temperature dependence of the modeled material properties and not a random result of slightly different optimization steps.

\section{Conclusion and Outlook}

The transducer model can be used to accurately describe the absolute electric impedance of transducers. Model parameters are determined in a multi-step identification process, resulting in only a minimal deviation of the simulated electric impedance from actual measurements. The results also exhibit physically plausible temperature dependence.

In further studies, the model will be validated using measurements of time-domain signals (i.e. electric send and receiving signals) and simulations of the transducer's impulse response. It will also be applied to more transducer variants, which are characterized by different resonance frequencies.

Afterwards, it is intended to make use of the measurement system by performing ultrasonic transmission measurements on a variety of samples. In the process, the model will be used to eliminate the transducer's influence on the measurement results.

\section{Acknowledgements}

The authors would like to thank the German Research Foundation (DFG) for financial support of the research project HE 2897/3-1 (determination of acoustic material properties).

\section{References}

[1] F. Bause, J. Rautenberg, B. Henning: Design, modeling and identification of an ultrasonic composite transducer for target impedance independent short pulse generation. AMA Conferences - Sensor, Nuremberg, 2013

[2] R. Lerch, G. Sessler, D. Wolf: Technische Akustik. Springer, 2009

[3] W. P. Mason: Electro-mechanical transducers and wave filters. Princeton: van Nostrand (1948)

[4] S. I. Kabanikhin: Inverse and III-Posed Problems: Theory and Applications. De Gruyter, 2012

[5] S. Haykin: Adaptive Filter Theory, Fourth edition. Prentice Hall, 2001

[6] C. H. Bischof, H. M. Bücker, B. Lang, A. Rasch, A. Vehreschild: Combining Source Transformation and Operator Overloading Techniques to Compute Derivatives for MATLAB Programs, Conference proceeding, Proceedings of the Second IEEE International Workshop on Source Code Analysis and Manipulation (SCAM 2002), IEEE Computer Society, 2002. 